\title{
The Diversity Dilemma
}

\section{John Staddon}

Diversity, not of ideas but of unrelated characteristics like race or ethnicity, is often assumed to imply intellectual diversity. But there are a couple of obvious constraints.

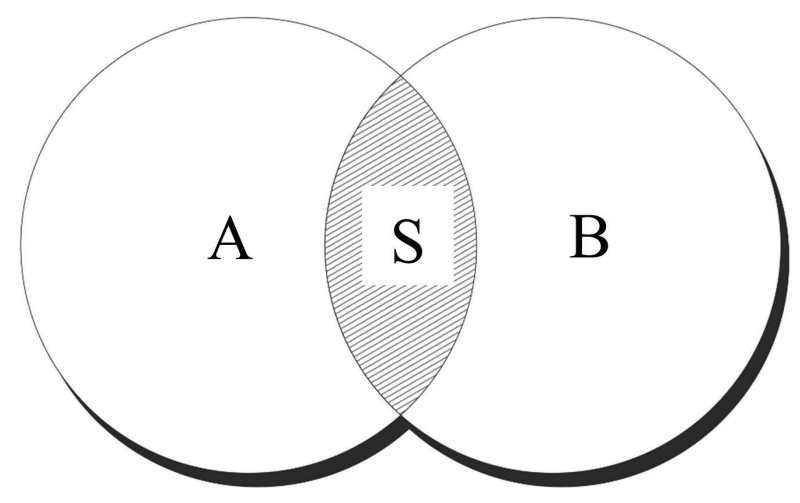

Any two different collections of individuals will have repertoires (sets of possible ideas) that differ as well as some ideas that will overlap, common to both groups. The diagram shows two such groups. The common repertoire of shared ideas is represented by S. The repertoires of A and B are represented by the circles. So, for these two groups, the combination will have a potential enlarged repertoire, $\mathrm{T}$, as follows:

$\mathrm{T}_{\mathrm{all}}=\mathrm{A}+\mathrm{B}-\mathrm{S}$, which represents an increase in idea diversity, so long as $\mathrm{S}<$ B.

John Staddon is James B. Duke Professor Emeritus, Department of Psychology and Neuroscience and Professor of Biology, Duke University. His most recent book is The New Behaviorism: Foundations of Behavioral Science 3rd Edition (Taylor and Francis, 2021). Staddon's article "The Devolution of Psychological Science: Memes, Culture, and Systemic Racism" appears in this issue of $A Q$. 


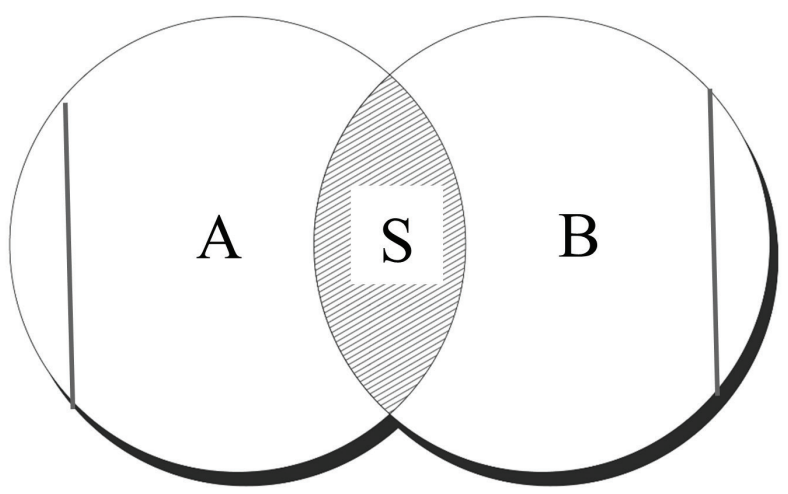

There is another constraint. Each group will have some ideas that are anathema to the other group, things that cannot be discussed by the combined group without discord. To ensure harmony, these repertoires must be excluded from discussion when the groups are combined. These troublesome ideas are represented by $A_{b}$ (A ideas unacceptable to $B$ ) and $B_{a}$ in the diagram. Thus, the total repertoire of the two groups combined, $\mathrm{T}_{\text {net }}$, will now be:

$$
\mathrm{T}_{\text {net }}=\mathrm{A}+\mathrm{B}-\left(\mathrm{S}+\mathrm{A}_{\mathrm{b}}+\mathrm{B}_{\mathrm{a}}\right)
$$

Diversity will represent an enlargement of the total repertoire, A, of the initial group only if

$$
\mathrm{T}_{\text {net }} \geq \mathrm{A} \text {, i.e., } \mathrm{B}>\left(\mathrm{S}+\mathrm{A}_{\mathrm{b}}+\mathrm{B}_{\mathrm{a}}\right)
$$

Two factors must therefore be balanced to maximize $\mathrm{T}_{\text {net }}$ : the groups should be as compatible as possible so as to minimize factors $A_{b}$ and $B_{a}$, which subtract from the total repertoire. But at the same time, the groups should be as different as possible so as to minimize overlap, S. These two requirements will often be in conflict if not incompatible with each other.

One can imagine various scenarios. For example, suppose that group B is much more sensitive than group A, meaning that many of A's ideas will be regarded as anathema by $\mathrm{B}$ and so must be excluded from the combined group. If group $A$ has a larger repertoire than group $B$, it is likely that $A_{b}$ will be large enough for $\left(A_{b}+B_{a}+S\right)>B$, so $T_{\text {net }}<A$, meaning that the addition of group $B$ 
actually reduces the repertoire diversity of the combined group to below that of A alone.

Conclusion: If the aim is to enlarge repertoire diversity by combining two groups, their sensitivities must be taken into account. If one group is easily offended, the combination is likely to be less intellectually diverse than either group on its own.

There is no guarantee that racially and ethnically diverse groups will yield greater intellectual diversity than a single homogeneous group. 